\title{
Monte Carlo Analysis of the SO(3) Lattice Gauge Theory and the Critical Dimensionality of Space-Time
}

\author{
M. Baig \\ Departament de Física Teórica, Universitat Autónoma de Barcelona, Bellaterra (Barcelona), Spain \\ (Received 8 August 1984)
}

\begin{abstract}
The analysis of the phase structure of the $\mathrm{SO}(3)=\mathrm{SU}(2) / \mathrm{Z}(2)$ lattice gauge theory in different space-time dimensionalities is presented. With use of Monte Carlo simulations the absence of phase transitions is shown for both $d=2$ and $d=3$. In the five-dimensional case I observe the persistence of the first-order phase transition previously seen at $d=4$. A monopole condensation mechanism is suggests as responsible for this transition.
\end{abstract}

PACS numbers: 11.15.Ha, 05.70.Fh, 11.30.Ly

The lattice gauge theories introduced by Wegner ${ }^{1}$ for the Abelian $\mathrm{Z}(2)$ gauge group and extended by Wilson $^{2}$ to the non-Abelian case are one of the most important ways to study nonperturbative properties of the quantum chromodynamics theory, such as confinement and hadron mass generation. Indeed, confinement appears as a general property of the strongcoupling regime of all lattice gauge theories. In lattice QCD the weak-coupling region connects with the continuum theory, occurring at $g$ (coupling) and $a$ (lattice spacing) equal to zero, the critical point representing asymptotic-freedom behavior. Near this point the theory scales according to the renormalization-group predictions. The transition region from strong coupling to weak coupling has received much attention. The reason is that the presence of a phase transition in this region may separate confinement from asymptotic freedom. One of the methods used to investigate this intermediate region is to look for phase transitions in Monte Carlo simulations. The absence of a phase transition in the four-dimensional SU(2) theory together with the existence of a second-order transition in the compact $\mathrm{U}(1)$ case (electrodynamics) gives strong support to the above picture. ${ }^{3}$

On the other hand, it has been recognized that there is a critical character of the space-time dimensionality in the presence of phase transitions. Indeed, Monte Carlo results suggest the presence of a first-order phase transition in the five-dimensional SU(2) theory ${ }^{4}$ and the absence of phase transitions in the threedimensional $\mathrm{U}(1)$ case. In consequence, $d=4$ can be considered as a critical dimensionality for the SU(2) lattice gauge theory while for the $\mathrm{U}(1)$ case it is $d=3$. In a general way, mean-field analysis predicts the presence of a first-order phase transition for all lattice gauge theories when the space-time dimensionality is high enough.

Although the four-dimensional SU(2) theory shows no phase transitions, there is a sharp peak in the specific heat precisely where the string tension shows a rapid crossover connecting the strong- and weakcoupling regimes. The dynamics of this intermediate region has received much attention. ${ }^{5}$ It is related to such topological configurations on the lattice as vortices and magnetic monopoles. In turn, these are related to the $\mathrm{Z}(2)$ center of the $\mathrm{SU}(2)$ gauge group. For this reason, the $\mathrm{SO}(3)=\mathrm{SU}(2) / \mathrm{Z}(2)$ theory that manifests $\mathrm{Z}(2)$ invariance has been investigated. Monte Carlo analysis shows the presence of a firstorder phase transition. ${ }^{6}$ In addition, Bhanot and Creutz ${ }^{7}$ working on a mixed SU(2)-SO(3) action, corresponding to mixed fundamental-adjoint representations, presented the complete phase diagram of the theory.

In this Letter the analysis of the phase structure of the SO(3) lattice gauge theory at different space-time dimensionalities is presented. Monte Carlo simulations and low-order strong- and weak-coupling developments have been used. The main results of the study are to show the absence of phase transitions for both $d=2$ and $d=3$ and the persistence at $d=5$ of the first-order phase transition previously seen at $d=4 .^{6}$

The SO(3) lattice gauge theory can be expressed in terms of the SU(2) matrices since it corresponds to the adjoint representation of the SU(2) group. The action is then

$$
S=\frac{1}{3} \beta\left(\operatorname{Tr}_{A} U_{p}\right)=\frac{1}{3} \beta\left(\left|\operatorname{Tr} U_{p}\right|^{2}-1\right),
$$

where $\operatorname{Tr} U_{p}$ means the trace of the ordered product of SU(2) matrices in the fundamental representation along an elementary square of the lattice. The numerical manipulations have been performed by use of the modified Metropolis algorithm with eight attempts per link. ${ }^{8}$ The extra phase transition due to the discreteness of the icosahedral approximation is close to the interesting region in the $\mathrm{SO}(3)$ theory and for this reason I have used the complete $\mathrm{SU}(2)$ group in all the computations.

Thermal cycles have been done at $d=2,3$, and 5 in order to detect hysteresis loops denoting the existence of phase transitions. The quantity measured has been the plaquette energy

$$
E=1-\frac{1}{3} \operatorname{Tr}_{A} U_{p} .
$$

I have used the lattice sizes $16^{2}, 10^{3}$, and $3^{5}$ except for 


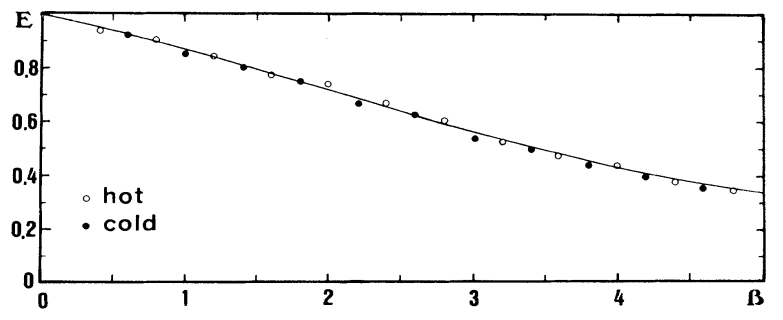

FIG. 1. Thermal cycle for the two-dimensional SO(3) lattice gauge theory. The continuous line represents the exact solution.

the intermediate region where additional sizes have also been used. Each point of the cycles represents an average over $20-30$ configurations after some twenty equilibrating iterations, starting on the final state of the previous point.

The strong-coupling expansion used is

$$
E=1-\beta / 9-\beta^{2} / 58+\beta^{4} / 1458,
$$

valid for all dimensionalities. The lowest-order weakcoupling expansion is, in turn,

$$
E=3 / \beta d,
$$

where $d$ is the space-time dimensionality.

The thermal cycle for $d=2$ is plotted in Fig. 1. For this two-dimensional (2D) case I do not find any evidence denoting the existence of phase transitions. Note that there is a very smooth transition between the strong- and weak-coupling phases. On the other hand, the 2D SO(3) lattice gauge theory is exactly solvable. In a similar way as in $\operatorname{SU}(N)$ or $\mathrm{U}(N)$ theories, ${ }^{9}$ it is possible to define a one-link integral for the partition function,

$$
Z(\beta)=\int_{G} d U \exp \left(\frac{1}{3} \beta \operatorname{Tr}_{A} U\right)
$$

Introducing in this expression the usual representation of the SU(2) matrices I obtain the following expression for the plaquette energy:

$$
E=1+\frac{1}{3} \frac{I_{2}(2 \beta / 3)-I_{1}(2 \beta / 3)}{I_{0}(2 \beta / 3)-I_{1}(2 \beta / 3)},
$$

where $I_{n}$ are the usual modified Bessel functions. This exact solution is also plotted in Fig. 1. The agreement with the Monte Carlo results is complete. This may be considered as a valuable test of the Monte Carlo accuracy.

The three-dimensional case shows an instability in the intermediate region. Figure 2 shows a thermal cycle using twenty iterations at each step. However, this hysteresis loop disappears when more iterations are performed at each step. Figure 3 displays the behavior of two long runs taking $\beta=3.3$, a value inside the hysteresis loop, with starting configurations cold and hot. Since the values after some fifty iterations coincide,

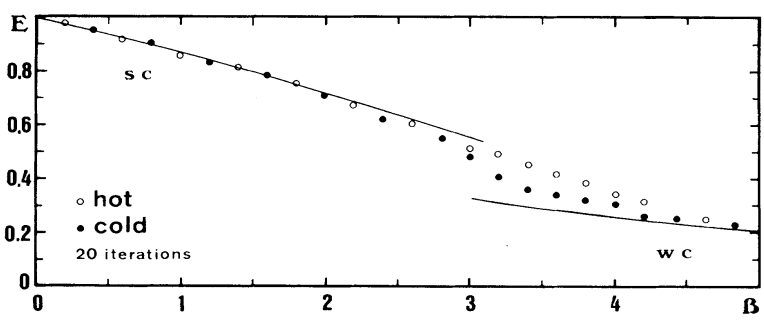

FIG. 2. Thermal cycle for the three-dimensional SO(3) lattice gauge theory.

these fluctuations do not correspond to a first-order transition. I feel that there are no phase transitions of higher order at this point. The convergence is too rapid for a second-order transition. Indeed, this behavior is similar to that observed in the fourdimensional SU(2) theory and it can be interpreted as the "shadow" of the transition present in more dimensions. To support this conclusion I have looked at the specific heat using direct numerical derivation of the energy,

$$
C=\beta^{2} d E / d \beta \text {. }
$$

Although there are large errors present when using this method of evaluation, I have no evidence for a peak increasing with the lattice size from $6^{3}$ and $12^{3}$ lattices. A second-order phase transition seems, then, to be excluded. Nevertheless, a high-order strongcoupling analysis of the fluctuations is in progress.

Figure 4 shows the results for the $5 \mathrm{D}$ case. Here I find a clear hysteresis loop near $\beta \simeq 2$. This suggests the existence of a phase transition. To study the order and precise location of this transition, I have done some Monte Carlo runs using a mixed ordereddisordered starting configuration. The results of these runs are collected in Fig. 5. This graph confirms that the transition is of first order and gives a value for the critical point of $\beta_{c}=2.08 \pm 0.01$. This value is very close to the modified mean-field prediction of Alberty, Flyvbjerg, and Lautrup ${ }^{10}$ which is $\beta_{c}=1.97 \pm 0.06$.

Finally, in order to determine the nature of the

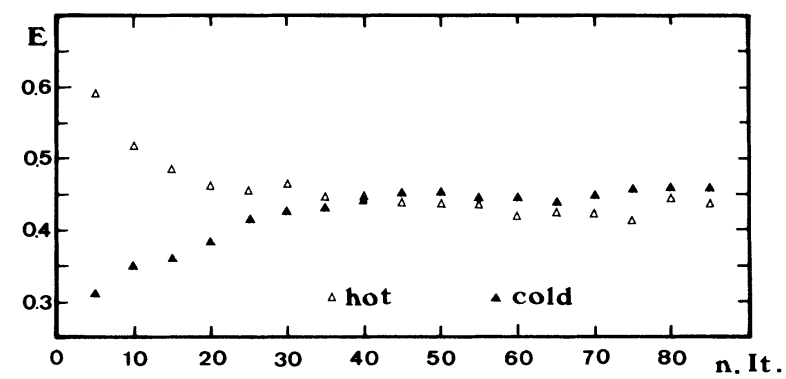

FIG. 3. Monte Carlo runs for the 3D SO(3) lattice gauge theory at $\beta=3.3$ with ordered and disordered starting points. 


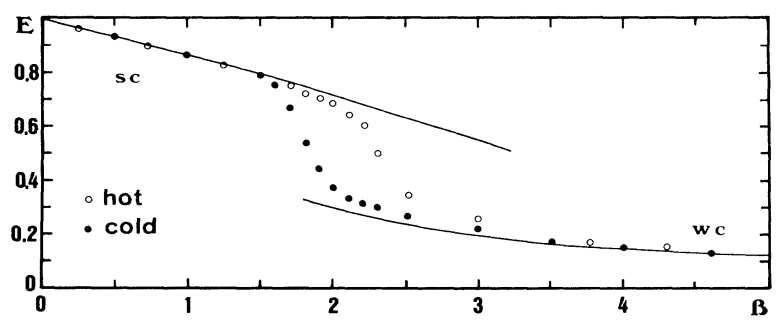

FIG. 4. Thermal cycle for the five-dimensional SO(3) lattice gauge theory.

phase transition seen at $d=5$, the monopole density is studied. Indeed, the first-order phase transition present at $d=4$ seems to originate from a monopole condensation. Explicit computations of the monopole density carried out by Haliday and Schwimmer ${ }^{6}$ using Villain's form of the action and by Mack and Pietarinen ${ }^{11}$ using the standard Wilson action showed a rapid decrease of the monopole density precisely at the phase transition point. A hysteresis cycle also appeared. Following Ref. 11 I define the parameter

$$
\rho_{c}(U)=\prod_{p \in \partial c} \operatorname{sgn} \operatorname{Tr} U(\partial p)= \pm 1 \in Z(2),
$$

i.e., the product of the signs of traces of the six plaquettes $(\partial p)$ belonging to a three-dimensional elementary cube $(\partial c)$. Cubes with $\rho_{c}(U)=-1$ denote the presence of a monopole in their interior. Note that the $Z(2)$ invariance of the SO(3) action implies that the average of negative-sign plaquettes is always equal to 0.5. Figure 6 shows the result of a Monte Carlo thermal cycle on a $3^{5}$ lattice, measuring the monopole density. A very clear condensation phenomenon appears in the transition region $\left(\beta_{c} \simeq 2.08\right)$. This result suggests that, as in the four-dimensional case, the first-order phase transition seen in the five-dimen-

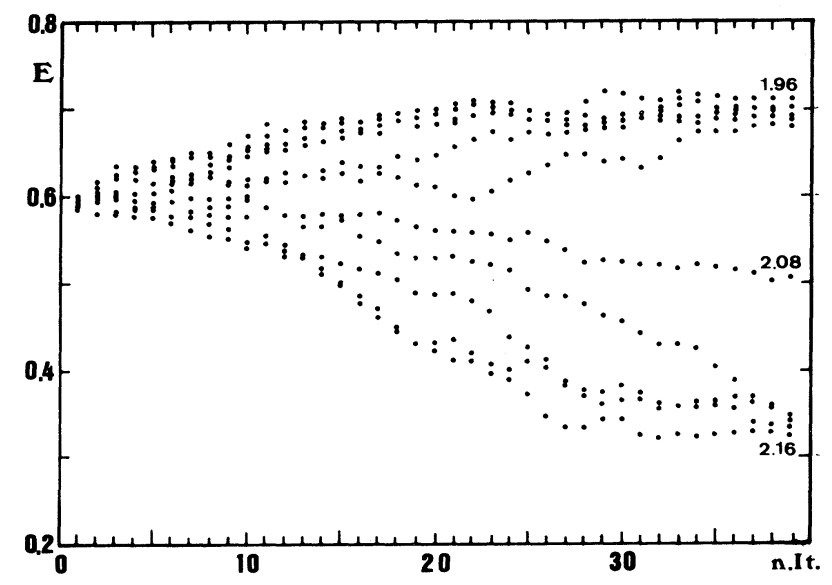

FIG. 5. Monte Carlo runs for the 5D SO(3) lattice gauge theory with a mixed ordered-disordered starting configuration. The step between $\beta$ values is 0.02 .

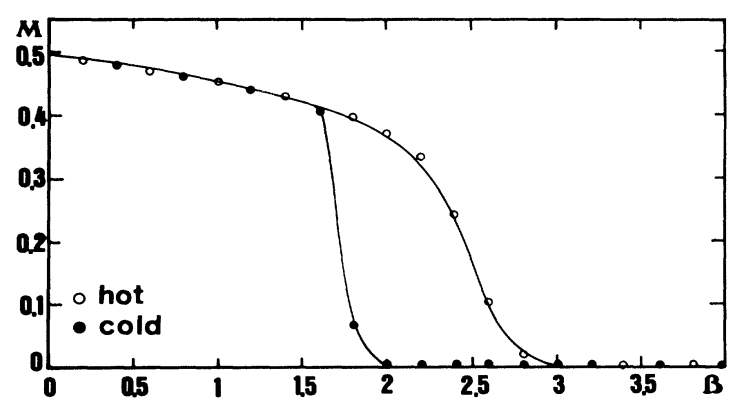

FIG. 6. Monte Carlo determination of the monopole density in the 5D SO(3) lattice gauge theory. The continuous lines are only for guiding the eye.

sional SO(3) lattice gauge theory originates from a monopole condensation mechanism.

In conclusion, I have determined, using Monte Carlo simulations, the absence of phase transitions, at least of first order, for both $d=2$ and $d=3$, and the persistence at $d=5$ of the first-order phase transition previously seen at $d=4$, with a critical $\beta_{c}$ $=2.08 \pm 0.01$. These results agree with the mean-field predictions of Ref. 10. A monopole condensation mechanism is suggested as responsible for this phase transition.

The author is grateful to Dr. C. Garcia Canal for useful discussions and remarks. The numerical computations have been performed on the VAX $11 / 780$ VMS computer of the Universitat Autónoma de Barcelona. This work was supported in part by Comísión Asesora de Investigación Científica y Técnica (Spain) through Project No. 0534/81.

${ }^{1}$ F. J. Wegner, J. Math. Phys. 10, 2259 (1971).

${ }^{2}$ K. Wilson, Phys. Rev. D 10, 2445 (1974).

${ }^{3}$ M. Creutz, Phys. Rev. D 21, 2308 (1980).

${ }^{4}$ B. Lautrup and M. Nauenberg, Phys. Lett. 95B, 63 (1980); M. Creutz, Phys. Rev. Lett. 43, 553 (1979).

${ }^{5}$ R. Brower, D. Kesler, and H. Levine, Phys. Rev. Lett. 47, 621 (1981); C. B. Lang, C. Rebbi, P. Salomonson, and B. S. Skagerstam, Phys. Lett. 101B, 173 (1981).

6I. G. Haliday and A. Schwimmer, Phys. Lett. 101B, 327 (1981); J. Greensite and B. Lautrup, Phys. Rev. Lett. 47, 9 (1981).

${ }^{7}$ G. Bhanot and M. Creutz, Phys. Rev. D 24, 3212 (1981); G. Bhanot and R. Dashen, Phys. Lett. 113B, 299 (1982).

${ }^{8}$ R. W. B. Ardill, K. J. M. Moriarty, and M. Creutz, Comput. Phys. Commun. 29, 97 (1983).

${ }^{9}$ C. B. Lang, P. Salomonson, and B. S. Skagerstam, Phys. Lett. 100B, 29 (1981).

${ }^{10}$ J. M. Alberty, H. Flyvbjerg, and B. Lautrup, Nucl. Phys. B220, (FS8), 61 (1983).

${ }^{11}$ G. Mack and E. Pietarinen, Nucl. Phys. B205 (FS5), 141 (1982) 